\title{
Sensitivity of cosmic-ray experiments to ultra-high-energy photons: reconstruction of the spectrum and limits on the superheavy dark matter
}

\author{
O.E. Kalashev, G.I. Rubtsov, and S.V. Troitsky \\ Institute for Nuclear Research of the Russian Academy of Sciences, \\ 60th October Anniversary Prospect 7a, Moscow 117312 Russia
}

\begin{abstract}
We estimate the sensitivity of various experiments detecting ultra-high-energy cosmic rays to primary photons with energies above $10^{19} \mathrm{eV}$. We demonstrate that the energy of a primary photon may be significantly (up to a factor of $\sim 10$ ) under- or overestimated for particular primary energies and arrival directions. We consider distortion of the reconstructed cosmic-ray spectrum for the photonic component. As an example, we use these results to constrain the parameter space of models of superheavy dark matter by means of both the observed spectra and available limits on the photon content. We find that a significant contribution of ultra-high-energy particles (photons and protons) from decays of superheavy dark matter is allowed by all these constraints.
\end{abstract}

PACS numbers: 98.70.Sa, 96.50.sbe, 96.50.sd 


\section{INTRODUCTION}

Recent studies [1, 2, 3, 4] put strong limits on the presence of photons among primary particles of ultra-high-energy (UHE, $\gtrsim 10^{19} \mathrm{eV}$ ) cosmic rays $(\mathrm{CR})$. However, while at energies $\sim 10^{19} \mathrm{eV}$ current gamma-ray limits ( $<2 \%$ of the integral flux of cosmic particles at $95 \%$ CL [4]) are very restrictive, the best limit at $10^{20} \mathrm{eV}$ allows (at 95\% CL) as much as 36\% of primary gamma rays [2]. At the same time, the reconstruction of the UHECR spectrum often relies on a general assumption of hadronic primaries. This assumption is explicit in Monte-Carlo simulations (AGASA [5], Telescope Array [6]) and implicit in methods which use calibration relations obtained for the bulk of the lower-energy events which are mostly hadronic (Yakutsk [7], Pierre Auger [8]). Well justified at $10^{19} \mathrm{eV}$, this approach may lead to systematic distortions of the spectrum in the very interesting energy range $\gtrsim 10^{20} \mathrm{eV}$, where a significant fraction of gamma rays is allowed. Because of this systematics, models which predict primary photons of these energies should be tested with great care.

The purpose of this paper is twofold. First, we allow for a hypothetical contribution of primary photons which is consistent with all experimental limits and study its possible effect on the derivation of the spectrum. Second, we consider particular theoretical models which predict such a contribution and constrain them with the simultaneous account of the spectrum and of photon limits. Though ultra-high energy photons have not been observed by now, they are expected to be seen among secondary products of the Greisen-ZatsepinKuzmin [9, 10] reaction (see e.g. Ref. [11]). They are also predicted in exotic hypothetical top-down models of UHECR origin (see Refs. [12, 13] for reviews), notably in the superheavy dark-matter (SHDM) models.

In this paper, we give a quantitative analysis of reconstruction of the spectrum by various experiments in the presence of a fraction of gamma-ray primaries. This analysis is obligatory when exotic scenarios of UHECR origin are constrained: theoretical predictions for the photon fraction depend on the normalization of the exotic contribution to the spectrum.

Air showers induced by primary photons differ significantly from the bulk of hadroninduced events (see e.g. Ref. [14] for a recent review). There are two competitive effects responsible for the diversity of showers induced by primary photons. First, due to the Landau, Pomeranchuk [15] and Migdal [16] (LPM) effect the electromagnetic cross-section is suppressed at energies $E>10^{19} \mathrm{eV}$. The LPM effect leads to the delay of the first 
interaction and the shower arrives to the ground level under-attenuated. Another effect is the $e^{ \pm}$pair production due to photon interaction with the geomagnetic field above the atmosphere. Secondary electrons produce gamma rays by synchrotron radiation generating a cascade in the geomagnetic field. The probability of this effect is proportional to the square of the product of photon energy and perpendicular component of geomagnetic field. The shower development therefore depends on both zenith and azimuthal angles of photon arrival direction. If the effect is strong enough, the particles enter the atmosphere with energies below the LPM threshold. As a result, not only the shower development differs from that of an average hadronic shower but also this difference is strongly direction-dependent.

The energy reconstructed by an experiment may therefore differ significantly from the true energy of the primary photon. In addition, acceptance of fluorescence detectors for photons may differ from that for hadronic primaries which is assumed in the spectral calculation. The difference in the reconstructed energy and acceptance should be accounted for individually for each experiment. In this note, we estimate quantitatively this difference in the energy reconstruction and discuss its possible effect on the spectrum and implications for constraining the SHDM models.

UHECR spectra measured by different experiments are not in the mutual agreement. The disagreement is sometimes attributed to systematic errors in energy determination. Both the normalization of AGASA [17], HiRes [18] and Yakutsk [19] spectra and the position of the astrophysically motivated dip agree within this approach [20]. The spectrum observed by the Pierre Auger Observatory (PAO) [21] agrees with the others in the region above $10^{19} \mathrm{eV}$ in the same assumption, see Fig. 1. The energy rescaling is motivated by discrepancies in different methods of energy estimation for hadronic showers: for instance, the energy estimated by the surface detector of PAO alone is about $30 \%$ larger than the energy estimated in the standard reconstruction procedure based on calibration to fluorescence-detector data [22]. For AGASA and Yakutsk the use of CORSIKA for energy estimation leads to systematic shifts of energies downwards by about $10-15 \%$ and $40 \%$, respectively [23, 24, 25]. Note that the rescaled spectra do not coincide at the highest energies $\left(E \gtrsim 10^{20} \mathrm{eV}\right)$; the discrepancy may be attributed either to insufficient statistics or to the presence of energydependent systematics, for instance of a non-standard component at the highest energies. This question should be addressed in the future with more data available.

Several limits on the fraction $\epsilon_{\gamma}$ of UHE photons in the integral cosmic-ray flux have 


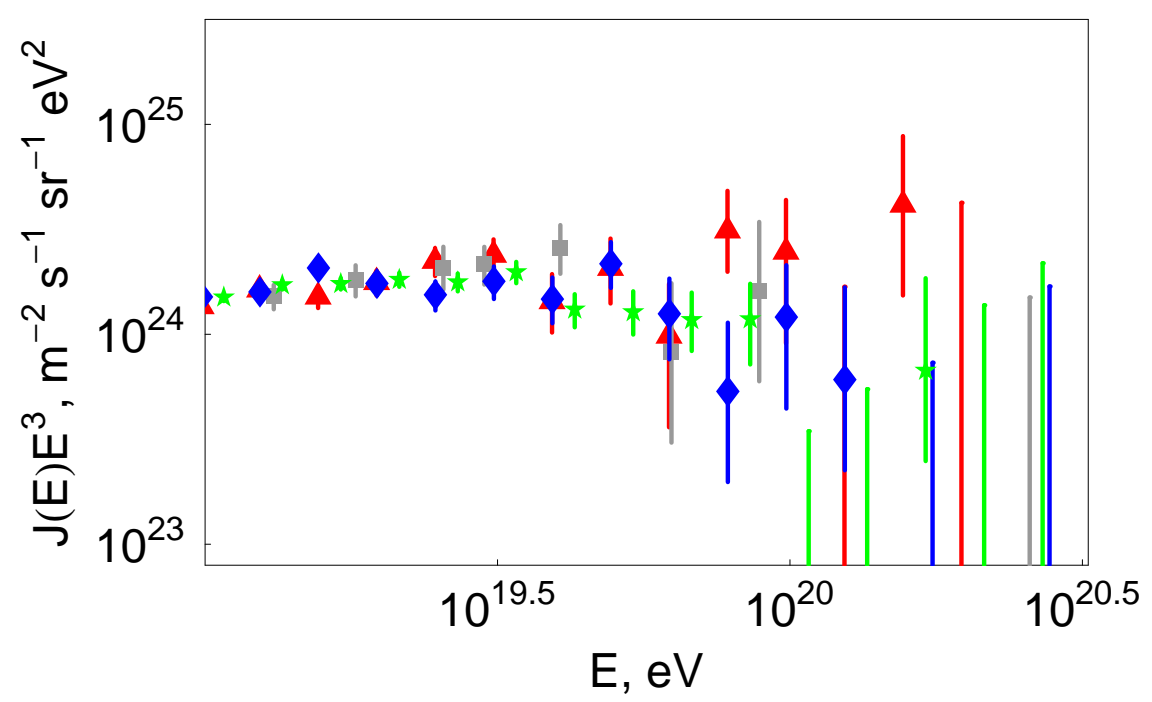

FIG. 1: Spectra of AGASA [17] (red triangles), HiRes [18] (blue diamonds), Yakutsk [19] (grey boxes) and PAO [21] (green stars) for energies scaled according to the best fit with protons from extragalactic sources described in Sec. III.

been set by several independent experiments. They are summarized in Fig. 2, The most restrictive limits (95\% C.L.) are $\epsilon_{\gamma}<0.36$ for $E>10^{20} \mathrm{eV}$ from the AGASA and Yakutsk joint dataset [2], $\epsilon_{\gamma}<0.22$ for $E>4 \times 10^{19} \mathrm{eV}$ from Yakutsk [3], $\epsilon_{\gamma}<0.05$ for $E>2 \times 10^{19} \mathrm{eV}$ and $\epsilon_{\gamma}<0.02$ for $E>10^{19} \mathrm{eV}$ from the Auger surface detector [4]. Even when the energy reconstruction of photons is properly taken into account (which was done in the calculation of these most restrictive limits), the limits on $\epsilon_{\gamma}$ may depend on the uncertainty in the energy reconstruction of non-photonic primaries, notably in the case of low statistics (see discussion and Fig. 2 in Ref. [3]). A more stable quantity is the flux of gamma rays; the Pierre Auger upper limit on the integral flux of photons above $10^{19} \mathrm{eV}$ is $3.8 \times 10^{-3} \mathrm{~km}^{-2} \mathrm{sr}^{-1} \mathrm{yr}^{-1}$ at the $95 \% \mathrm{CL}$. These photon limits may be used to constrain top-down models provided a theoretical model for the top-down photon flux is given. In a self-consistent analysis, the latter should be normalized to the observed spectrum. This normalization requires in turn the account of the energy reconstruction of photons which constitute a significant fraction of the top-down flux. Below, we perform a joint analysis of the spectrum and of photon limits for the SHDM models and constrain the space of two SHDM parameters (mass and lifetime of the superheavy particle). Contrary to the previous studies, most of which used either a naive AGASA normalization or an overall (independent from the energy and arrival 


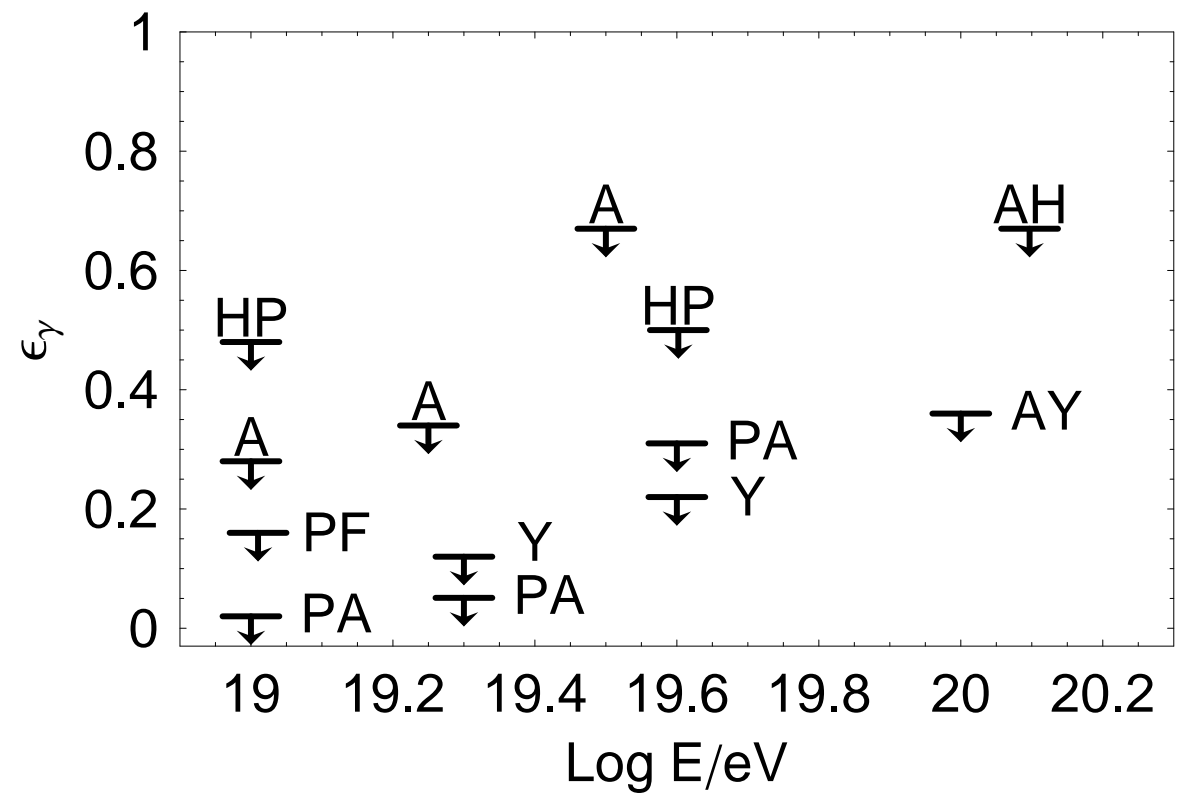

FIG. 2: Upper limits (95\% C.L.) for the fraction $\epsilon_{\gamma}$ of gamma rays in the integral flux of cosmic rays with energies higher than E: Haverah Park [26] (HP), AGASA 27] (A), 11] (AH), AGASA and Yakutsk [2] (AY), Yakutsk [3] (Y), Auger fluorescence detector [28] (PF), Auger surface detector [4] $(\mathrm{PA})$.

direction) multiplicative correction for the reconstructed photon energies, our results suggest that a significant fraction of cosmic rays from the SHDM decays is allowed by all experimental constraints.

The rest of the paper is organized as follows. In Sec. II we estimate sensitivity of four experiments (AGASA, HiRes, Pierre Auger and Yakutsk) to the primary photon component. In Sec. III we consider an example of constraining SHDM parameters using primary spectra and photon limits. Section [IV summarizes our results.

\section{SENSITIVITY TO THE PHOTON COMPONENT}

To calculate the spectrum of photons reconstructed by a given experiment it is important to account both for the energy estimation of a particular photon and for the experiment's exposure to photons. We obtain approximate estimates in the following way:

$A G A S A$. The array has a geometrical exposure for hadronic primaries with energies above $10^{18.5} \mathrm{eV}$. The probability to accept an event by the ground detector depends only on the 
detector signal which is, for a given arrival direction, in one-to-one correspondence with the reconstructed energy of the event. Therefore, the exposure is geometrical for photons with reconstructed energies above $10^{18.5} \mathrm{eV}$. To calculate reconstructed energies for primary photons we run Monte-Carlo simulations using CORSIKA 6.611 [29] with GHEISHA [30] as a low-energy hadronic interaction model and EPOS 1.61 [31] as a high-energy hadronic model. Since the hadronic component carries a small fraction of energy of a photon-induced shower, dependence of the results on the choice of hadronic model is negligible within our precision. EGS4 [32] is used to model electromagnetic interactions and the PRESHOWER code [33] is used to account for possible interactions of the primary photons with the geomagnetic field. The reconstructed energy for primary photons is calculated by means of the standard AGASA procedure [5] using the detector response obtained from the GEANT simulations in Ref. [34]. The results of the analysis are presented in Fig. 3, left column. Photon-induced showers penetrate deeply and are therefore younger when they reach the surface detector, as compared to the hadronic ones. This fact results in overestimation of the primary energy because of a bias in the attenuation correction (which was fit to the bulk of - presumably hadronic - showers by means of the constant intensity cuts method). On average, energies of showers with $E>10^{19} \mathrm{eV}$ are overestimated by a factor of $\sim 2$, but for particular energies and arrival directions where the geomagnetic cascade does not compensate the LPM suppression, the overestimation may reach a factor of ten. We note that in Ref. [2], energies of individual highest-energy AGASA events have been estimated within the primary-photon assumption, so the corresponding limit on the gamma-ray fraction does not suffer from this problem.

HiRes. The exposure of the HiRes fluorescent detector for the primary photons was calculated in Ref. [35] and has been found to be almost twice smaller than the exposure for protons. The reason is the reduced efficiency of reconstruction of deep showers, so that a significant part of them does not pass strict quality cuts determined for the spectrum-related studies (e.g. the maximum of the shower development fully seen). Energies reconstructed by the fluorescence method for different primary particles differ only by the contribution of particles not taking part in the electromagnetic cascade. The correction is calculated in Ref. [36]; its application for the gamma-ray showers results in primary photons energy overestimation by about $10 \%$, well within the systematic uncertainties. 

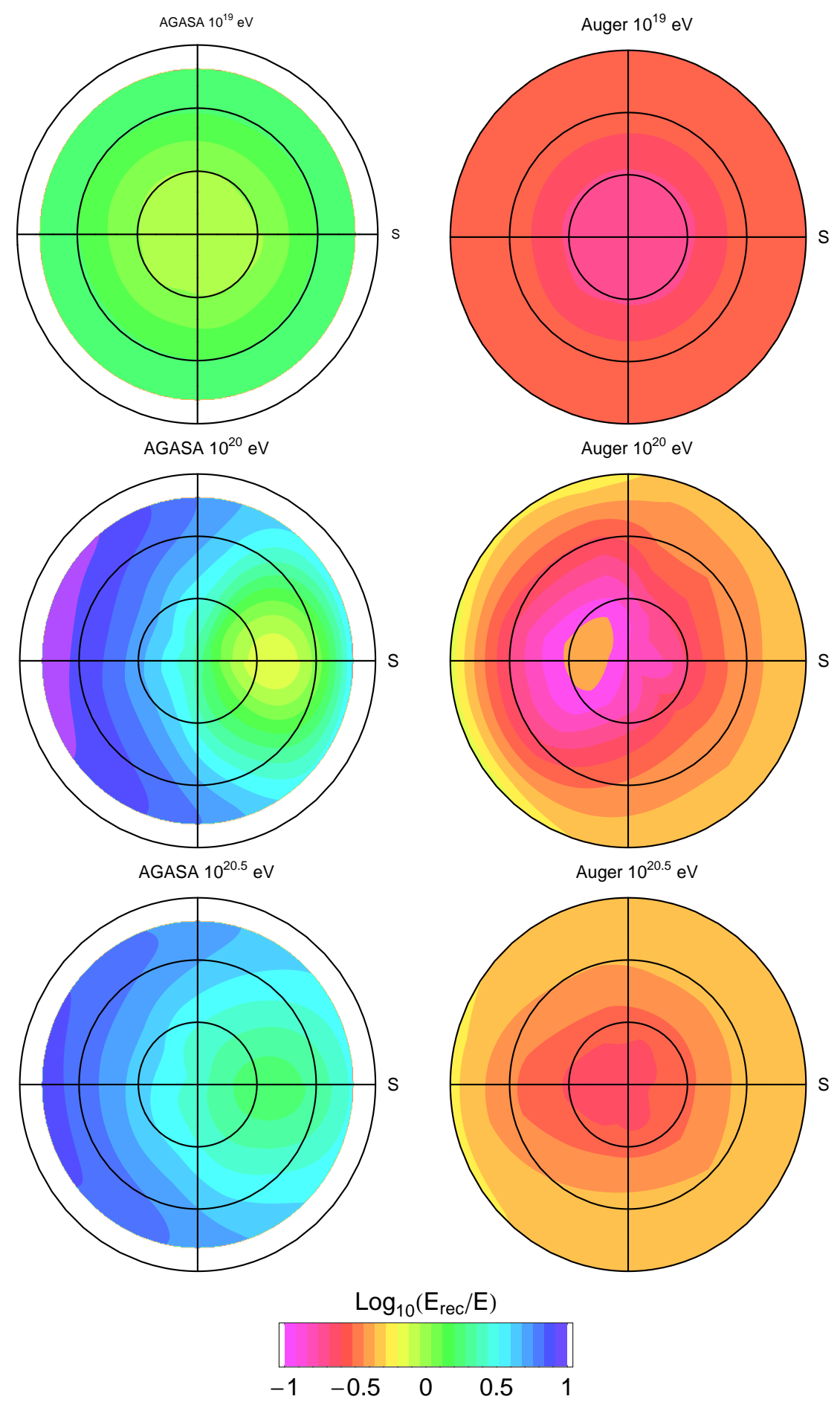

FIG. 3: Energy overestimation factor for photon showers observed by AGASA (left column) and by the surface detector of PAO as a function of energy (shown for three values marked on the plots) and arrival direction (radial coordinate: zenith angle, angle coordinate: azimuth; zenith is in the center and South is to the right of the plots). The logarithmic colour code is shown in the bottom panel. 
Pierre Auger. The surface detector of PAO also has the geometrical exposure at the highest energies we are interested in. The detector response of Auger water tanks is not publicly available and therefore we use $S(1000)$ values for photon-induced showers without geomagnetic preshower from Fig. 3 of Ref. [37]. We separately perform preshower simulations using the CORSIKA PRESHOWER module for El Nihuil location and use data from Ref. [37] for secondary photons. Finally we reconstruct the primary energy using formulae of Ref. [8]. The results of our analysis are presented in Fig. 3, right column. It turns out that the photon primary energies are underestimated (for the spectrum derivation) by the PAO surface detector by the factor of four in average. The underestimation may reach an order of magnitude for particular energies and arrival directions. The physical reason for the photon energy underestimation is hypersensitivity of the water tanks to the muon component of the shower, while the latter is strongly suppressed in photon-induced showers. A completely different energy reconstruction procedure, which assumes primary gamma rays, has been applied [4] for the calculation of the photon limits. The latters are therefore insensitive to this problem.

Yakutsk. The exposure of the Yakutsk EAS array is also geometrical. The spectrum below $10^{19} \mathrm{eV}$ is obtained using a small subarray [19], so the exposure depends on the energy in a known way. To obtain reconstructed energies for the primary photons we use the Monte-Carlo simulations (similar to those described above for AGASA) and the Yakutsk detector response obtained from GEANT simulations in Ref. [38]. Qualitatively, the results are very similar to those obtained for AGASA. The gamma limits of Refs. [2, 3] have been calculated with the account of the energy reconstruction for primary photons.

The results of the analysis for various experiments are illustrated in Figs. 3, 4.

With the statistics presently available it is not possible to explain the difference in spectra at the highest energies by means of the photon component. Our consideration nevertheless illustrates that the presence of a non-standard component might influence the interpretation of experimental results.

\section{CONSTRAINTS ON THE SHDM PARAMETERS}

As an example of application of our results, we study how the systematics in the determination of the spectrum in the presence of primary photons may affect constraints on the 


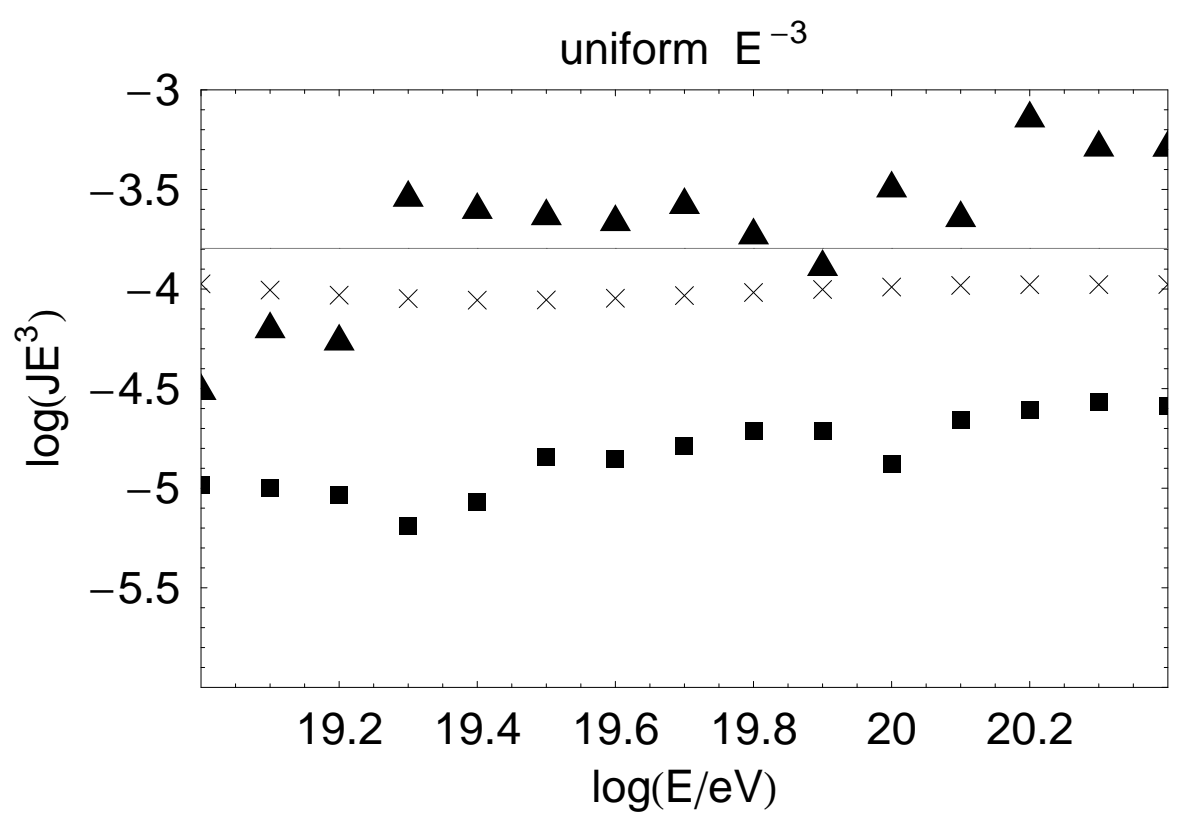

FIG. 4: The spectra reconstructed by different experiments (triangles: AGASA, crosses: HiRes, boxes: Auger) for thrown isotropic photon flux $E^{-3}$ (gray line).

SHDM obtained from the limits on the primary gamma rays.

The SHDM models predict very hard spectrum with a large fraction of photons and therefore both the spectral shape and gamma limits can be used to constrain the models. We perform a joint fit of spectra of four experiments above $10^{19} \mathrm{eV}$ with the sum of astrophysical and SHDM components and obtain constraints on the parameters of SHDM model. The full spectral fit is performed as described below and $95 \%$ C.L. limits on the gamma-ray content listed in Sec. [I] are imposed as theta-functional constraints. Since some of the gamma limits are given in terms of the fraction, and not of the absolute flux, of primary photons, they cannot be analysed independently of the spectra. For the photon component, we use the spectrum reconstruction for each experiment as described above, while for hadronic primaries, we consider the energy scale as a parameter of fit individual for each experiment, see details below. We take into account both photons and protons produced in SHDM decays.

The astrophysical contribution. We simulate propagation of cosmic rays from astrophysical sources using the numerical code [39] with a few modifications described in Ref. [40].

The code uses the kinematic-equation approach and calculates the propagation of nucleons, stable leptons and photons [57] using the standard dominant processes (as is explained, 
for instance, in Ref. [12]). For nucleons, it takes into account single and multiple pion production and $e^{ \pm}$pair production on the CMB, infrared/optical and radio backgrounds, neutron $\beta$-decays and the expansion of the Universe. For photons, the code includes $e^{ \pm}$ pair production, $\gamma+\gamma_{B} \rightarrow e^{+} e^{-}$and double $e^{ \pm}$pair production $\gamma+\gamma_{B} \rightarrow e^{+} e^{-} e^{+} e^{-}$, processes. For electrons and positrons, it takes into account inverse Compton scattering, $e^{ \pm}+\gamma_{B} \rightarrow e^{ \pm} \gamma$, triple pair production, $e^{ \pm}+\gamma_{B} \rightarrow e^{ \pm} e^{+} e^{-}$, and synchrotron energy loss on extragalactic magnetic fields (EGMF). The propagation of the electron-photon cascade and nucleons are calculated self-consistently, namely secondary particles arising in all reactions are propagated alongside the primaries. The hadronic interactions of nucleons are derived from the well established SOPHIA event generator [41].

UHE particles lose their energy in interactions with the photon background, which consists of CMB, radio, infra-red and optical (IRO) components, as well as EGMF. Protons are sensitive essentially to the CMB only, while for UHE photons and nuclei the radio and IRO components are respectively important. Although the radio background is not yet well known our conclusions do not depend strongly on the radio background assumed since secondary photons from proton propagation are in any case subdominant and their flux remains below the present limits (see Ref. [40] for more details; for SHDM the effect is also not important because the SHDM flux is dominated by the Milky-Way contribution). We use estimates by Clark et al. [42] for extragalactic radio background and model of Ref. [43] for the IRO background component. The IRO background is only important to transport the energy of secondary photons in the cascade process from the $0.1-100 \mathrm{TeV}$ energy range to the 0.1-100 GeV energy range observed by EGRET, and thus the resulting flux in the energy range of our interest is not sensitive to details of the IRO background models.

We assume pure proton composition at injection and take the spectrum of an individual UHECR source to be of the form:

$$
F(E)=f E^{-\alpha} \Theta\left(E_{\max }-E\right),
$$

where $f$ provides the flux normalization, $\alpha$ is the spectral index and $E_{\max }\left(E_{\max }\right)$ is the maximum energy to which protons can be accelerated at the source.

We assume the standard cosmological model with the Hubble constant $H=$ $70 \mathrm{~km} \mathrm{~s}^{-1} \mathrm{Mpc}^{-1}$, the dark energy density (in units of the critical density) $\Omega_{\Lambda}=0.7$ and a 
matter density $\Omega_{\mathrm{m}}=0.3$. We define total source density in this model as

$$
n(z)=n_{0}(1+z)^{3+m_{z}} \Theta\left(z_{\max }-z\right) \Theta\left(z-z_{\min }\right)
$$

where $m_{z}$ parameterizes the source density evolution, in such a way that $m_{z}=0$ corresponds to non-evolving sources with constant density per comoving volume, and $z_{\min }$ and $z_{\max }$ are respectively the redshifts of the closest and most distant sources. In this paper we use a fixed value of $z_{\max }=3$.

The SHDM contribution. Decays of the SHDM particles may be described in a more or less model-independent way because the most important physical phenomenon of relevance is hadronization which involves light particles and is well understood. Denote $x \equiv \frac{2 E}{M_{X}}$, where $E$ is the energy of a decay product of the SHDM particle with mass $M_{X}$. Then for $10^{-4} \lesssim x \lesssim 0.1$, spectra of the decay products calculated by various methods [44, 45] are in a good agreement with each other; moreover, the shape of the spectral curve $\frac{d N}{d E}(x)$ does depend only mildly on $M_{X}$ [44] and we may consider the dependence negligible. For this study, we use the spectra of decay products from Ref. [44].[58]

The SHDM decay rate is determined by the concentration $n_{X}$ and lifetime $\tau_{X}$ of the SHDM particles, $\dot{n}_{X}=n_{X} / \tau_{X}$. The flux of secondary particles at the Earth is then determined by

$$
j=\mathcal{N} \frac{1}{\tau_{X}} \frac{d N}{d E}
$$

where

$$
\mathcal{N}=\int d^{3} r \frac{n_{X}(\mathbf{r})}{4 \pi r^{2}}
$$

is the geometrical factor ( $\mathbf{r}$ is the radius-vector from the Earth; though in principle one should integrate over the Universe and account for relativity effects, in most interesting cases the dominant contribution comes from the Galactic halo [46]). The mass $M_{X}$ is subject to cosmological limits (see e.g. Ref. [47] and references therein); the lifetime $\tau_{X}$ is much less restricted.

The flux is assumed to be a sum of two components, one of which corresponds to the "bottom-up" contribution while the second one is due to the SHDM decays. While the former is assumed to be isotropic, the latter is not because of non-central position of the Sun in the Milky Way; we account for this anisotropy as described in Ref. [48], assuming the Navarro-Frank-White [49] dark-matter distribution and with obvious account of the 


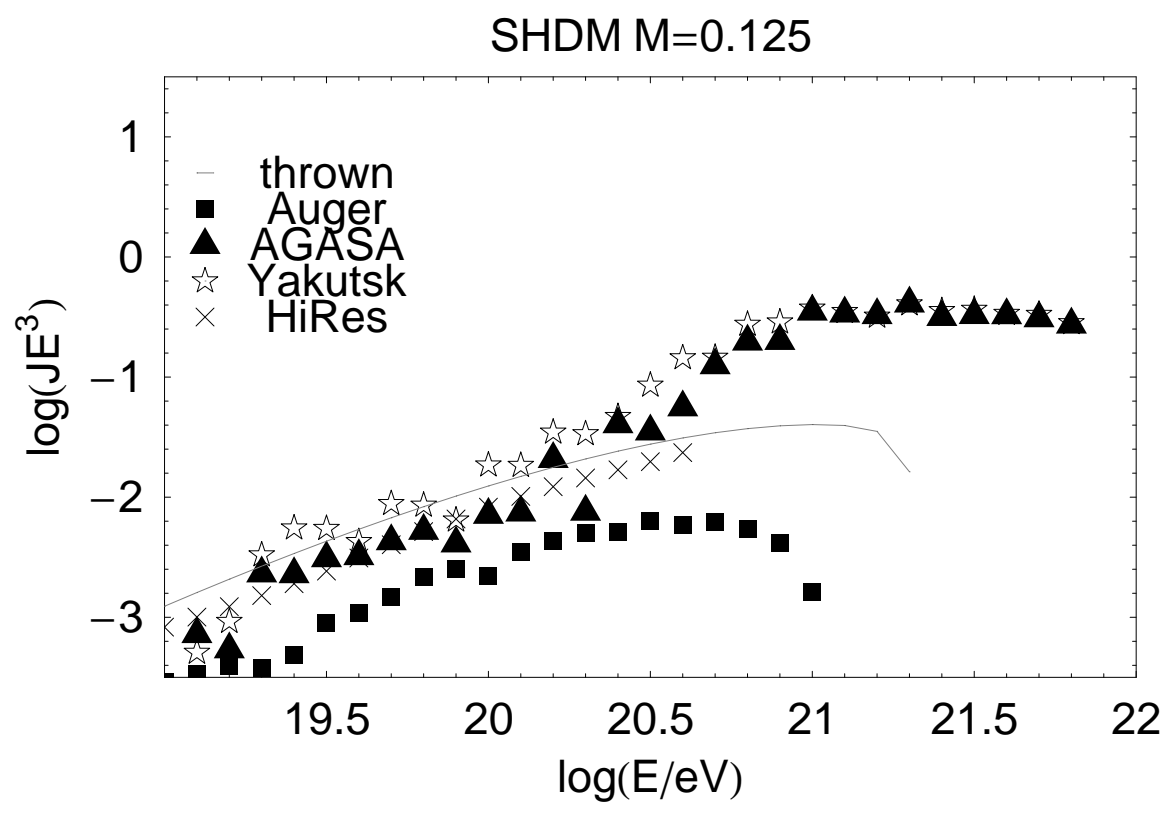

FIG. 5: Same as in Fig. 4 but for the initial photon spectrum predicted by decays of SHDM with $M_{X}=1.25 \times 10^{21} \mathrm{eV}$.

exposure (field of view) of particular experiments. The account of the anisotropy reduces the difference in the reconstructed spectra for the SHDM-related photons because the energy underestimation by PAO is partially compensated by the larger flux of photons seen in the Southern hemisphere, with an opposite effect for AGASA, see Fig. 5.

The fitting procedure. Up to the normalizations (depending on $\tau_{X}$ for the dark-matter part), the spectra are determined by four parameters $\left(\alpha, E_{\max }, m_{z}\right.$ and $\left.z_{\min }\right)$ for the astrophysical part and by $M_{X}$ for the SHDM part. We scan over these parameters which are let to take their values on a grid. For the astrophysical spectrum, we use the grid described in Ref. [40]; for $M_{X}$ we allow values $2^{k} \times 10^{22} \mathrm{eV}$ for seven integer values of $k$, $-3 \leq k \leq 3$. For each point on the five-dimensional grid, we fit four experimental energy spectra (AGASA [17], Yakutsk [19], HiRes [18] and PAO [21]) with four independent energy shifts representing energy-independent systematic errors of the four experiments and with two overall normalization factors (for the astrophysical and for the dark-matter parts), allowing these six parameters to change continuously.

We fit binned numbers of events detected by four experiments using the analog of $\chi^{2}$ for the Poisson data described e.g. in Ref. [50]. Technically, potential systematic errors of the energy determination of hadronic primaries (fit parameters) are taken into account as shifts 


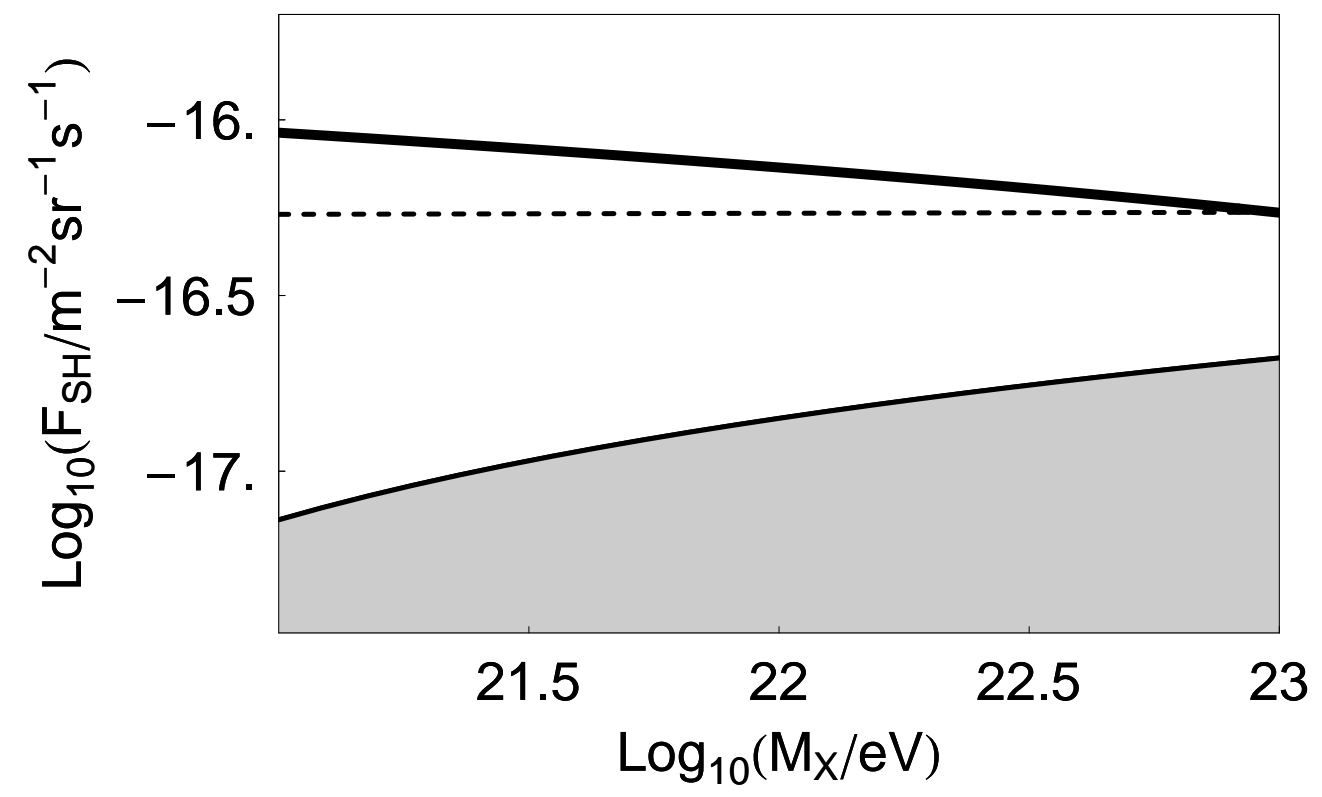

FIG. 6: Parameter space of the SHDM models: the total integral flux $F_{\mathrm{SH}}$ of the cosmic rays from SHDM decays at energies $E>10^{20} \mathrm{eV}$ (inversely proportional to the lifetime $\tau_{X}$ of the SHDM particle in particular models) versus the mass $M_{X}$ of the SHDM particle. The area above the thick line is excluded by the spectral fits; the area above the dashed line is excluded by the limit on $\epsilon_{\gamma}$ at $E>10^{20} \mathrm{eV}[2]$; the area above the thin line is excluded by the limit on the gamma-ray flux at $E>10^{19} \mathrm{eV}$ [4]. The shadowed area is allowed by any constraints.

of the theoretical curve and not of the data. We fix the experiments' exposure and do not fix the total number of the detected events.

Statistical errors in energy estimation are taken into account as described in Ref. [40]. They are assumed to be Gaussian in logarithmic scale with widths 25\%, 20\%, 6\% and 17\% for AGASA, HiRes, PAO and Yakutsk respectively [7, 17, 18, 21].

The goodness of fit is determined by the Monte-Carlo simulations as described in Ref. [51]. We consider a fit as acceptable if its goodness exceeds 0.05.

Results. Resulting constraints on the SHDM parameters from the spectral fits and photon limits are presented in Fig. 6. The spectral fits are equally good for models with and without SHDM [59] and a significant part of the SHDM parameter space does not contradict the observed spectra. As one might expect, the photon limits are more restrictive, but our analysis demonstrates that, contrary to the common lore, even they do not exclude 
AGASA
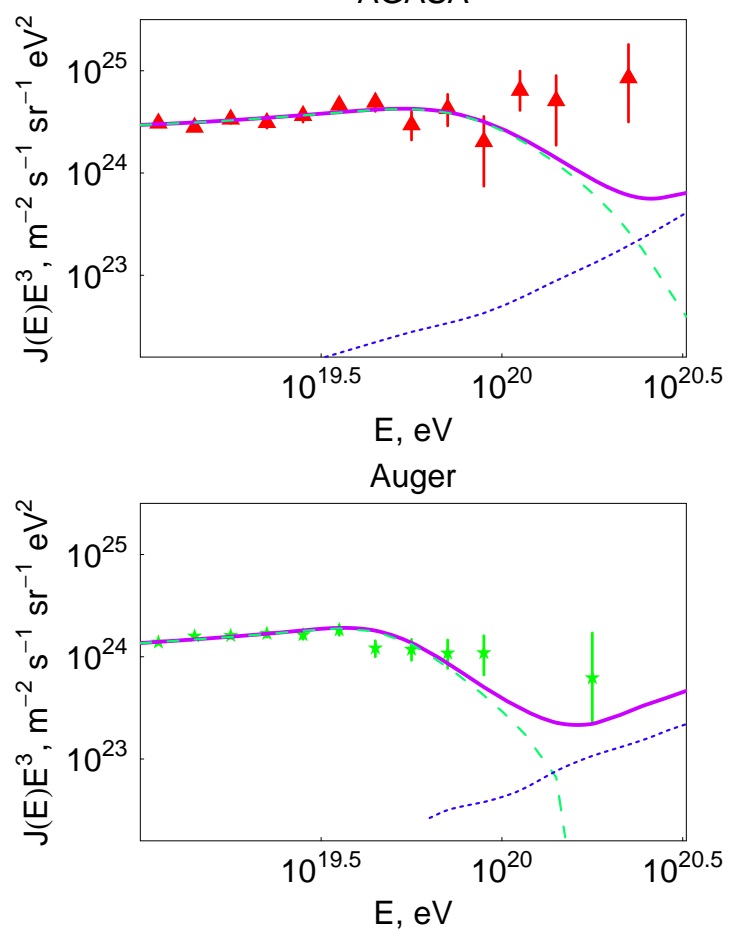
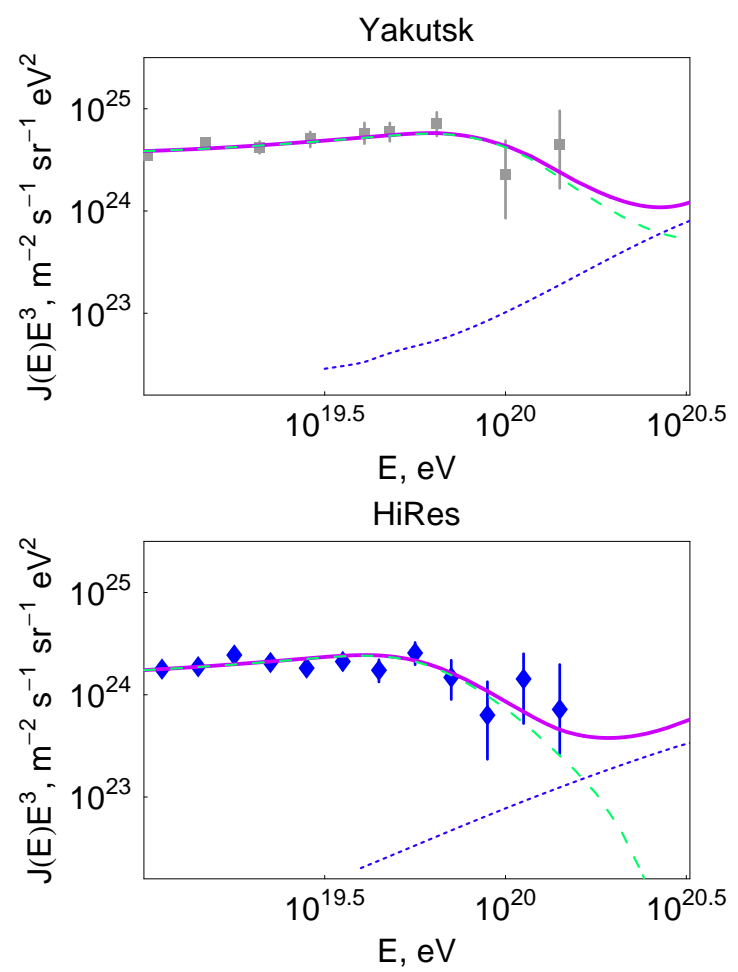

FIG. 7: The fit of the spectra observed by different experiments with the "extragalactic plus SHDM" model for which the SHDM contribution is maximal but all photon limits are satisfied. Symbols with error bars represent experimental data points, thick lines represent the fit, dashed lines - the extragalactic component of the fit function, dotted lines - contribution of photons from the SHDM decays. One and the same physical spectrum looks different for different experiments because the energy reconstruction is taken into account: for the hadronic component it is encoded in the energy shifts - parameters of the fit; for the gamma-ray component it is obtained explicitly in Sec. II

SHDM for a wide range of parameters: more than one half of the cosmic-ray particles with $E \gtrsim 10^{20} \mathrm{eV}$ may have their origin from the SHDM decays without violating any of the experimental constraints. In Fig. 7, we present gamma-ray and hadron spectra for one of the models with maximal allowed SHDM contribution (similar spectra are obtained for several models with slightly different parameters of the astrophysical contribution). 


\section{CONCLUSION}

Modern experiments have different sensitivities to the photon component and this should be taken into account when testing particular models. The AGASA experiment overestimated the energy of primary photons with energies $E \gtrsim 10^{19} \mathrm{eV}$ by a factor of 2 in average, though the overestimation reaches a factor of $\sim 10$ for particular energies and arrival directions. Contrary, the surface detector of the Pierre Auger Observatory underestimates the energy of primary photons in this energy range by a factor of $\sim 4$ in average, while underestimation by a factor of $\sim 10$ happens for particular energies and arrival directions. The HiRes detector overestimated the photon energies by a factor of $\sim 1.1$, uniformly over arrival directions and well within the systematic uncertainties. However, it had a significantly lower exposure for primary photons than for primary hadrons.

One of the scenarios predicting a significant amount of primary UHE photons is the superheavy-dark-matter model. We analyzed constraints on its parameters from the observed spectra and limits on the photon content. While the most restrictive photon limits [2, 3, 4] account for peculiarities in the energy reconstruction for photons, a dedicated study was required and performed for the spectral fits. A significant (more than one half of the flux at $E>10^{20} \mathrm{eV}$ ) SHDM component is still allowed by all limits. Though there seems no present need for the SHDM to explain the UHECR spectrum, a large contribution of SHDM at the highest energies is not excluded and may be further constrained (or validated) by future experiments. Among the tests are measurements of the spectrum, studies of anisotropy and searches for primary photons and neutrinos. Our study indicates that a significant SHDM contribution is allowed for masses of dark-matter particles $M_{X} \gtrsim 4 \times 10^{22} \mathrm{eV}$, so one needs experiments with large aperture at $E>10^{20} \mathrm{eV}$ (e.g. JEM-EUSO [52]) to test the shape of the spectrum. At lower energies $\left(10^{19} \mathrm{eV}\right.$ to $\left.10^{20} \mathrm{eV}\right)$ the model may be constrained by improving the gamma-ray limits and by precise studies of the Galactic anisotropy, e.g. with the help of fluorescent detectors (which treat uniformly both photon and hadron primaries).

As a final remark, we note that the example of photons should warn one against naive tests of models predicting "exotic" primaries with the experimental data. For instance, the correlations with BL Lac type objects observed by HiRes [53, 54] require neutral primary particles. If the latters were photons, apparent absence of correlations in the preliminary 
data of the PAO surface detector [55] is easily explained [56] by underestimation of their energies as compared to the bulk of hadronic primaries. With more exotic primary particles, the analysis becomes even less trivial.

We are indebted to V. Berezinsky, D. Gorbunov, J.-M. Frére, M. Libanov, M. Pravdin, V. Rubakov, D. Semikoz, P. Tinyakov and I. Tkachev for valuable discussions. This work was supported in part by the Russian Foundation of Basic Research grants 07-02-00820 and 0907-00388, by Federal Agency for Science and Innovation under state contracts 02.740.11.0244 and 02.740.11.5092, by the grants of the President of the Russian Federation NS-1616.2008.2, MK-1966.2008.2 (OK), MK-61.2008.2 (GR), by the Dynasty Foundation (GR), by the Russian Science Support Foundation (GR), by IISN and by the Belgian Science Policy (IAP VI-11). Numerical part of the work was performed at the computer cluster of the Theoretical Division of INR RAS. GR and ST thank Service of Theoretical Physics, ULB, for kind hospitality.

[1] M. Risse et al., Phys. Rev. Lett. 95, 171102 (2005).

[2] G. I. Rubtsov et al., Phys. Rev. D 73, 063009 (2006).

[3] A. V. Glushkov et al., JETP Lett. 85, 131 (2007).

[4] J. Abraham et al. [Pierre Auger Collaboration], Astropart. Phys. 29, 243 (2008).

[5] M. Takeda et al., Astropart. Phys. 19, 447 (2003).

[6] A. Taketa et al. [Telescope Array Collaboration], Proc. 31st ICRC (Lodz), 2009, paper 0855.

[7] M.I. Pravdin, Proc. 29th ICRC (Pune), 2005.

[8] M. Roth [Pierre Auger Collaboration], arXiv:0706.2096 [astro-ph].

[9] K. Griesen, Phys. Rev. Lett. 16, 748 (1966)

[10] Z. T. Zatsepin and V. A. Kuz'min, Zh. Eksp. Teor. Fiz. Pis'ma Red. 4, 144 (1966)

[11] G. Gelmini, O. Kalashev and D. V. Semikoz, Astropart. Phys. 28, 390 (2007).

[12] P. Bhattacharjee, G. Sigl, Phys. Rept. 327, 109 (2000).

[13] M. Kachelriess, Comptes Rendus Physique 5, 441 (2004).

[14] M. Risse and P. Homola, Mod. Phys. Lett. A 22, 749 (2007).

[15] L. D. Landau and I. Ya. Pomeranchuk, Dokl. Acad. Nauk SSSR, 92, 535, 735 (1953)

[16] A. B. Migdal, Phys. Rev. 103, 1811 (1956) 
[17] M. Takeda et al., Astropart. Phys. 19, 447 (2003).

[18] R. U. Abbasi et al. [High Resolution Fly's Eye Collaboration], Phys. Rev. Lett. 92, 151101 (2004).

[19] V. P. Egorova et al., Nucl. Phys. Proc. Suppl. 136, 3 (2004).

[20] V. Berezinsky, A. Z. Gazizov and S. I. Grigorieva, Phys. Rev. D 74, 043005 (2006) arXiv:hep-ph/0204357.

[21] J. Abraham et al. [Pierre Auger Collaboration], Phys. Rev. Lett. 101, 061101 (2008).

[22] R. Engel [Pierre Auger Collaboration], arXiv:0706.1921 [astro-ph].

[23] M. Teshima for AGASA collaboration Proc. of 33th ICHEP, Moscow, 2006 http://ichep06.jinr.ru/reports/49_1s4_16p30_Teshima.ppt

[24] L. G. Dedenko et al., Nucl. Phys. Proc. Suppl. 165, 27 (2007).

[25] L. G. Dedenko, D. A. Podgrudkov, T. M. Roganova and G. F. Fedorova, arXiv:0811.0722 [astro-ph].

[26] M. Ave, J. A. Hinton, R. A. Vazquez, A. A. Watson and E. Zas, Phys. Rev. Lett. 85, 2244 (2000).

[27] K. Shinozaki et al., Astrophys. J. 571, L117 (2002).

[28] J. Abraham et al. [Pierre Auger Collaboration], Astropart. Phys. 27, 155 (2007).

[29] D. Heck et al., Report FZKA-6019 (1998), Forschungszentrum Karlsruhe.

[30] H. Fesefeldt, PITHA 85/02.

[31] K. Werner, F. M. Liu and T. Pierog, Phys. Rev. C 74 (2006) 044902.

[32] W. R. Nelson, H. Hirayama, D.W.O. Rogers, SLAC-0265 (permanently updated since 1985).

[33] P. Homola et al., Comp. Phys. Comm. 173 (2005) 71.

[34] N. Sakaki et al., Proc. ICRC 2001, 1, 333.

[35] V. de Souza, G. Medina-Tanco and J. A. Ortiz, Phys. Rev. D 72, 103009 (2005).

[36] T. Pierog et al, Proc. 29h ICRC, Pune 7, 103-106 (2005)

[37] P. Billoir, C. Roucelle and J. C. Hamilton, arXiv:astro-ph/0701583.

[38] L. G. Dedenko et al., Nucl. Phys. Proc. Suppl. 136, 12 (2004).

[39] O.E. Kalashev, V.A. Kuzmin and D.V. Semikoz, astro-ph/9911035. Mod. Phys. Lett. A 16, 2505 (2001); O.E. Kalashev Ph.D. Thesis, INR RAS, 2003.

[40] G. B. Gelmini, O. Kalashev and D. V. Semikoz, JCAP 0711, 002 (2007).

[41] A. Mucke, R. Engel, J. P. Rachen, R. J. Protheroe and T. Stanev, Comput. Phys. Commun. 
124, 290 (2000).

[42] T. A. Clark, L. W. Brown, and J. K. Alexander, Nature 228, 847 (1970).

[43] F. W. Stecker, M. A. Malkan and S. T. Scully, Astrophys. J. 648, 774 (2006).

[44] R. Aloisio, V. Berezinsky and M. Kachelriess, Nucl. Phys. Proc. Suppl. 136, 319 (2004).

[45] S. Sarkar and R. Toldra, Nucl. Phys. B 621, 495 (2002); C. Barbot and M. Drees, Phys. Lett. B 533, 107 (2002); C. Barbot and M. Drees, Astropart. Phys. 20, 5 (2003).

[46] S. L. Dubovsky and P. G. Tinyakov, JETP Lett. 68, 107 (1998).

[47] D. J. H. Chung, E. W. Kolb, A. Riotto and L. Senatore, Phys. Rev. D 72 (2005) 023511.

[48] O. E. Kalashev, B. A. Khrenov, P. Klimov, S. Sharakin and S. V. Troitsky, JCAP 0803, 003 (2008).

[49] J. F. Navarro, C. S. Frenk and S. D. M. White, Astrophys. J. 462, 563 (1996).

[50] S. Baker and R. D. Cousins, Nucl. Instrum. Meth. 221, 437 (1984).

[51] W. H. Press et al. "Numerical Recipes in C: The Art of Scientific Computing", Cambridge University Press (1992)

[52] A. D. Supanitsky et al., arXiv:0909.1302 [astro-ph.HE].

[53] D. S. Gorbunov, P. G. Tinyakov, I. I. Tkachev and S. V. Troitsky, JETP Lett. 80 (2004) 145.

[54] R. U. Abbasi et al. [HiRes Collaboration], Astrophys. J. 636 (2006) 680.

[55] D. Harari [The Pierre Auger Collaboration], arXiv:0706.1715 [astro-ph].

[56] S. V. Troitsky, Mon. Not. Roy. Astron. Soc. 388 (2008) L79.

[57] In the present study, we do not use the possibility of heavier nuclei primaries which is allowed by the code.

[58] We thank M. Kachelrieß for providing numerical tables of the functions caculated there.

[59] The best fit without SHDM has a goodness of 0.19 and corresponds to energy scaling factors of $0.92,1.04,0.70$ and 0.60 for HiRes, PAO, AGASA and Yakutsk respectively (astrophysical model $z_{\min }=0, m_{z}=4, \alpha=2.45, E_{\max }=1.28 \times 10^{21} \mathrm{eV}$ ). The best fit with SHDM (which satisfies all photon limits) has a goodness of 0.25 and corresponds to an SHDM model with $M_{X}=2.5 \times 10^{21} \mathrm{eV}$ and energy shifts $0.95,1.07,0.72$ and 0.61 for HiRes, PAO, AGASA and Yakutsk respectively (astrophysical model $z_{\min }=0, m_{z}=4, \alpha=2.45, E_{\max }=6.4 \times 10^{20} \mathrm{eV}$ ). At (true) energies higher than $10^{20} \mathrm{eV}$, the SHDM-related component (photons and hadrons) comprises $43 \%$ of the total cosmic-ray flux for the best fit parameters. 